\title{
3D dynamic thermography system for biomedical applications
}

\author{
by G. Chernov*, V. Chernov**, C. Dávila-Peralta***, R. Rodriquez-Carvajal*** and M. Barboza- \\ Flores** $^{* *}$
}

\author{
* Departamento de Física, Doctorado en Nanotecnología, Universidad de Sonora, Hermosillo, Sonora, 83000, \\ México,g.chernovch@gmail.com \\ ** Departamento de Investigación en Física, Universidad de Sonora, Hermosillo, Sonora, 83000, México \\ *** Departamento de Ingeniería Industrial, Universidad de Sonora, Hermosillo, Sonora, 83000, México
}

\begin{abstract}
In this work we present a system for 3D dynamic thermography that consists of two FLIR thermal cameras and two off-the-shelf depth sensors. To generate a single 3D thermogram, the data obtained from the depth sensors is registered with the images from the thermal cameras. The process of generating a 3D thermogram is repeated several times while thermally stimulating the surface of the subject to produce a series of $3 \mathrm{D}$ thermograms. The advantage of this system is presence of $3 \mathrm{D}$ geometric data that is used to register the thermograms in the series to compensate for any movement of the subject and select regions of interest based on the subject's anatomy and easy segmentation of the body from the background.
\end{abstract}

\section{Introduction}

Infrared thermography, as a method for functional diagnostics, is gaining more and more traction in various fields of medicine, science and clinical practice. Medical thermography is an imaging technique that is fast, non-contact and radiation free that has proven useful for detecting and monitoring thermal abnormalities at the skin surface [1], that has been applied with some success at detecting many clinical conditios, for example: breast cancer [2], melanoma [3] and diabetic foot [4].

Standard thermograms, obtained using conventional infrared cameras, are two-dimensional in nature. One peculiar property of current infrared cameras is the effect of the distance and surface curvature on the reported temperature, as the angle between the viewing axis and the normal of the studied surface increases, the apparent temperature of the surface decreases [5]. This significantly limits the precision of the measurement of surface temperature on objects of complex shapes, such as different parts of the human body, which limits the applicability of thermography as a tool for medical diagnostics. To overcome these limitations currently there are in development several methods for obtaining 3D thermograms by combining polygonal meshes describing the complex surface with 2D infrared images. The presence of additional spacial information would allow such systems to calculate the viewing axis and the normals of the surface and to correct any errors due to the surface curvatures or positioning of the studied object. Currently there are several systems for acquisition of 3D thermograms described in the literature, most of them consisting of two distinct components, one for obtaining a 3D shape and one for obtaining an IR image, which are then merged together to form a 3D thermogram. The main difference between the proposed methods is the technique used for the acquisition of the 3D shape, each with unique advantages and disadvantages.

One kind of system for 3D thermogram acquisition is composed of a pair of RGB cameras and an IR camera $[6,7]$. The two RGB cameras are used to reconstruct the 3D shape using stereo imaging algorithms while the IR camera acquires a 2D thermogram. This method is susceptible to errors due to lighting conditions and the texture of the target object, but has the advantage of being relatively cheap to implement. Another approach to 3D image acquisition is a method based on structured light $[8,9,10]$, which involve projecting known patterns unto the target object and calculating its shape based on the distortion of the pattern. A third approach to obtaining 3D thermograms consists of using stereo vision $3 D$ reconstruction directly with IR cameras $[11,12]$. Finally, an approach that has been gaining popularity in recent years is the usage of off-the-shelf depth cameras for obtaining the 3D shape directly from the depth map produced by these devices [13, 14].

A depth camera is a device that produces an image in which the value of each pixel is proportional to the distance from the camera to the object at that pixel location. In recent years, a variety of such devices has become available commercially at a relatively low cost, for example the Microsoft Kinect and the Softkinetic DS325. These devices provide an accurate, but somewhat noisy, depth image that can be used for 3D reconstruction and to produce 3D thermograms with relatively little setup. These systems also have the advantage of having a small, self contained package that aids portability.

In addition to the usual passive method of thermography, dynamic thermography has been implemented as a valuable extension of the static thermography [15]. Dynamic thermography is based on the introduction of an external thermal stimulation and the subsequent observation of the thermal recovery process of the area of interest. This provides additional information about the distribution of heat flow and the thermal processes in the area below the surface.

In this work we present a 3D dynamic infrared thermography system that allows us to generate a series of 3D thermograms while thermally stimulating the surface of human body. 


\subsection{1/qirt.2016.020}

\section{4D thermography system}

The system consists of structural elements and sliding and rotating joints that allow the placement of cameras and sensors in space around the subject, at fixed positions and angles. Two FLIR thermal cameras and two depth sensors were used for the image acquisition and the construction of 3D thermograms. The depth sensors were rigidly mounted to the thermal cameras and their sensors were aligned, forming a single unit as seen in the inset photograph in figure 1 . Two such units are mounted on the arms of the device and turned to face the subject of the study. Using two sets of cameras ensures that the subject is visible from both sides simultaneously (figure 1).

The choice of depth sensors for this application is of particular importance, since it needs to meet two specific criteria: the acquisition speed must be fast and the sensors must not interfere with each other, since they must operate simultaneously. In particular on the second point, a sensor like the Microsoft Kinect V1 uses structured light with a nearinfrared projector and two such sensors operating at the same time would cause interference and return false data. A pair of Creative Senz3D time-of-flight sensors were chosen, due to the fact that their principle of operation is more resistant to interference from another sensor [16].

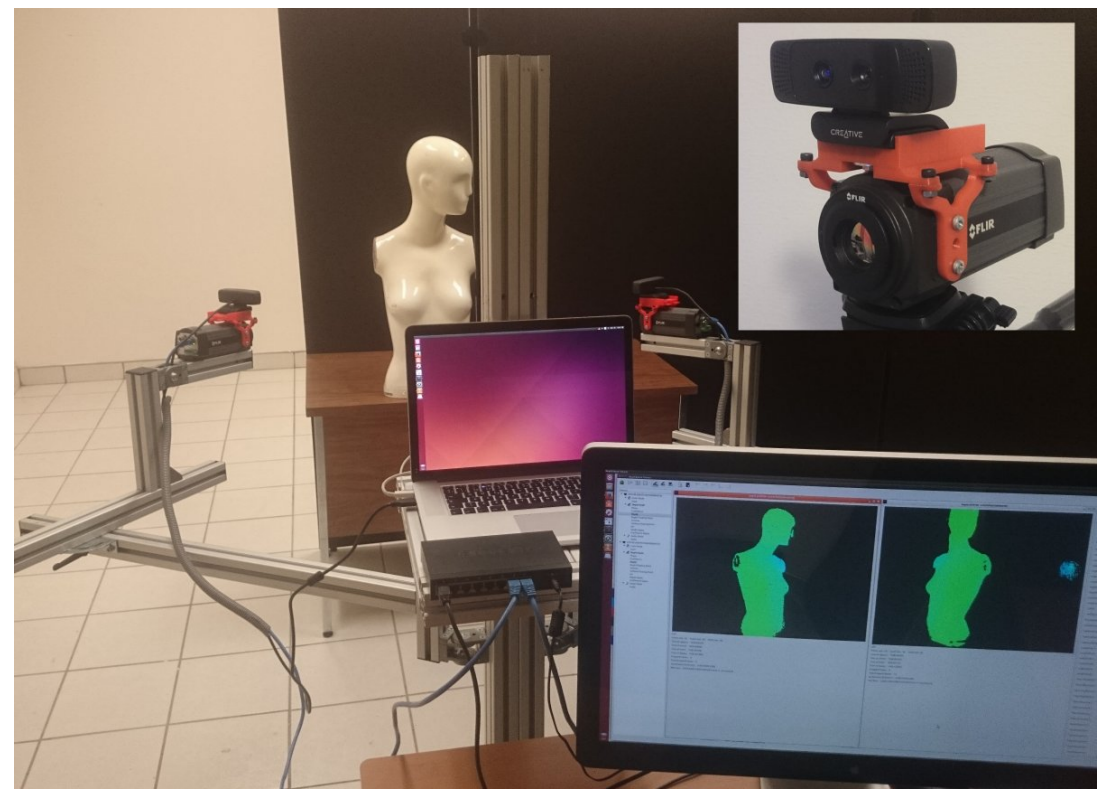

Fig. 1: Photograph of the 4D thermography system with two camera units mounted on rotating arms around a subject, each camera unit (inset photograph) consists of a FLIR A320 thermal imaging camera and a Creative Senz3D depth sensor.

\section{Software}

The software of the 4D thermography system is responsible for the data acquisition, processing and the construction of dynamic 3D thermograms. During the data acquisition phase, the raw thermal and depth images are downloaded from the connected sensors. Additionally, the raw data is often noisy and may have missing values, which means that the data must be filtered to remove as much noise as possible and compensate for any missing data. Once the quality of the incoming data is ensured, the next phase of the process consists of the transformation of the distance information obtained from the depth sensors into a point cloud in 3D space and the generation of a surface based on this point cloud, as well as the projection of the thermal images onto this surface to obtain a 3D thermogram. To perform this step, it is essential to know the physical parameters, such as focal lengths and distortion coefficients, of the cameras used and their locations in space relative to each other, for which a robust calibration method is required. This calibration only needs to be performed once when the system is set up, and then can be stored for future use.

To obtain a dynamic 3D thermogram, these steps are repeated however many times necessary while the surface of the subject is thermally stimulated. Once the dynamic 3D thermogram is obtained, it can then be processed and analyzed to obtain the dynamic thermal profile of the subject under study.

\subsection{Calibration and pose estimation}

To construct a $3 D$ thermogram, it is essential to have information about the position of the thermal cameras relative to the depth sensor, thus it is necessary to perform camera calibration. Camera calibration usually consists of two distinct steps. The first step is the calculation of intrinsic camera parameters, the focal lengths and distortion coefficients, 


\subsection{1/qirt.2016.020}

and it must be performed individually for each camera type used. The second step is the calculation of extrinsic camera parameters, the rotation and position of the camera in space, and it must be performed simultaneously for all the cameras used. The transformation matrices of the cameras must be calculated relative to a known point in space, the natural choice being one of depth cameras.

Camera calibration is performed using a calibration target. A calibration target consists of an object with features arranged in a known pattern and easily detectable using computer vision algorithms. Particular care must be taken when choosing a calibration target for our application, because the target needs to be visible and recognizable both on the depth image and the thermal image. A flat target with a pattern consisting of an asymmetric grid of circular holes of a fixed diameter works well for this purpose [14]. The calibration target was cut with a laser from $5 \mathrm{~mm}$ foamboard and mounted on a tripod (figure 2).

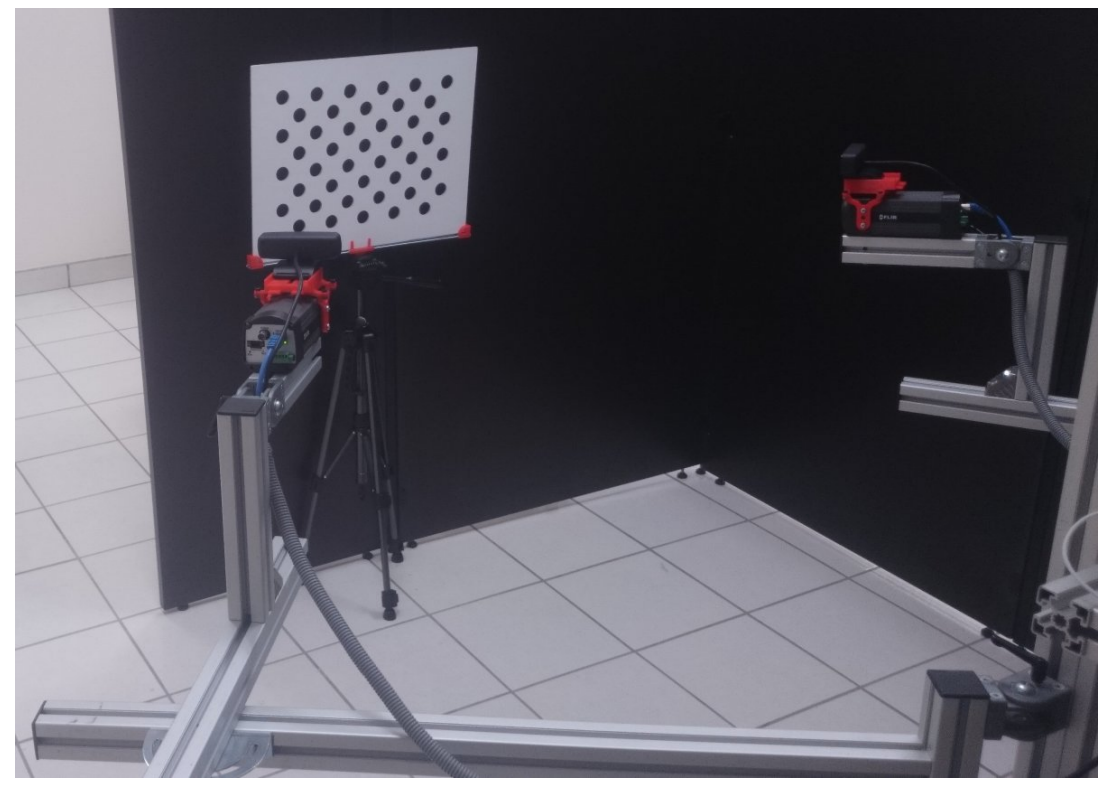

Fig. 2: Calibration setup used to find the intrinsic and extrinsic parameters of the camera system.

Camera calibration for the entire system was performed in separate steps. First, the intrinsic parameters of the thermal imaging cameras were calculated, individually for each camera, using images of the calibration targed rotated at various angles (figure 3. Intrinsic calibration of the Creative Senz3D was not necessary since the information was provided with the camera's documentation. Once the intrinsic parameters of all the cameras involved are obtained, the next step is to find the relative transformations between the depth camera and the thermal camera of one camera unit. This is done by pointing the camera unit towards a calibration target and estimating the position of the target on the image, using the previously obtained intrinsic parameters of each camera (figure 4). The final step necessary to fully calibrate the system is to obtain the locations of both camera units in relation to the subject. The procedure employed is similar to the calibration of a single camera unit, both units are pointed towards a single calibration target. Since the relative positions of all the cameras within a unit are known, it is enough to calculate the relative position of any one of the cameras in the unit to know the positions of the others, in this step of the calibration, only the depth cameras were used. All calibrations were done using a standard computer vision library, OpenCV [17].

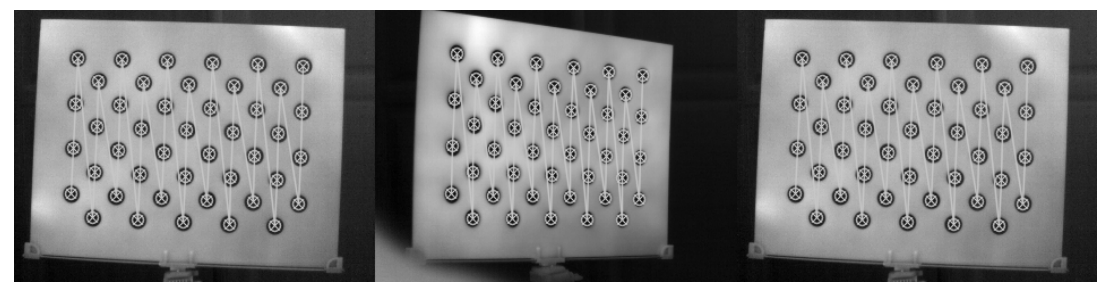

Fig. 3: Example images of the calibration target taken with a FLIR A320 camera used for finding intrinsic parameters of the camera. 

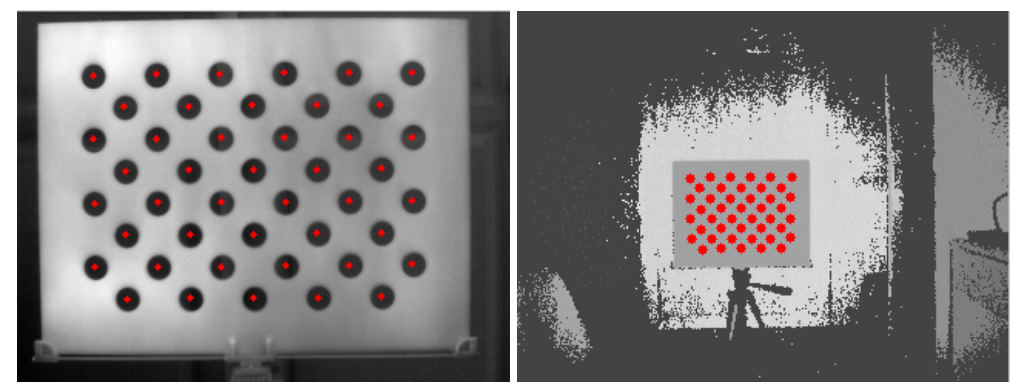

Fig. 4: Calibration target as seen on the thermal image (left) and the depth image (right). Red dots represent the locations of the hole centers in the calibration target as detected by the software.

\subsection{Data acquisition}

The system consists of four cameras, two of them are thermal cameras and two of them are depth cameras. In order to build a 3D thermogram at a particular instant in time, and image must be taken by each of the four cameras and transferred to a computer where the 3D thermogram can be assembled. It is important to ensure that the four images are taken as close as possible in time in order to provide adequate synchronization between depth and thermal images. The FLIR A320 cameras used stream their images over the network in an RTSP stream. Each thermal image consists of an array of 16-bit wide unsigned integers that linearly correspond to the temperature of the target object. The Creative Senz3D cameras connect via standard USB 2.0 and the data is obtained using an SDK provided by SoftKinetic for their DepthSense cameras. The Senz3D cameras provide a stream of depth images. Each depth image consists of an array of floating point numbers that correspond to the distance in millimeters to the object in that position.

The thermal images provided by the FLIR A320 cameras (figure 5) are useable as is and don't require further processing. On the other hand, depth images provided by modern time-of-flight sensors are somewhat noisy and require some cleanup. The noise is reduced by taking multiple depth images in succession and averaging them, and then applying a bilateral filter to the result [18]. This treatment improves the quality of the depth image and makes it more useful for surface reconstrution. As a final step, the depth images are transformed into a point cloud using the intrinsic parameters of the depth camera in preparation for the surface reconstruction.

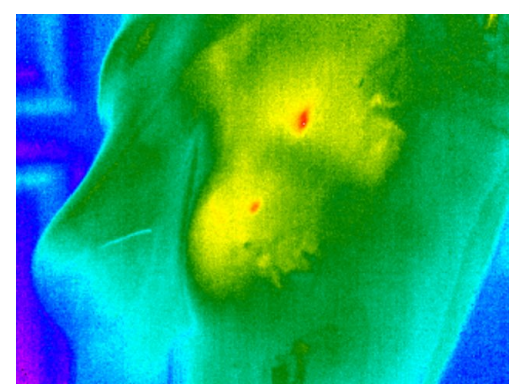

Fig. 5: Example of a thermal image output by the FLIR A320 thermal camera.

\subsection{Surface reconstruction}

After the data from the depth cameras and thermal cameras is obtained, cleaned and transformed to a point cloud, there still remains the problem of reconstructing the surface of the subject and assigning the correct temperature value to each point of the reconstructed surface. The first step in reconstructing the surface is to determine which points belong to the subject and which points do not. In this particular case, this is achieved by means of a simple distance threshold, since the subject is closer to the camera than any background points, thus we can confidently say that any points farther away from the depth camera than a certain distance do not belong to the subject. While this simple process eliminates most of the background, there may be several outlier points which are closer to the camera than the threshold but still don't belong to the subject. Points like these are removed using the tools provided by the PCL library [19] for removal of statistical outliers. The point clouds are then aligned into their correct positions using the information obtained in the camera calibration step and merged into one. The surface of the subject is the calculated from the point cloud data using the moving least squares method for surface reconstruction [20]. After the surface reconstruction is complete, what remains is to calculate the temperature at each point of the surface, this process is straightforward. Each point of the surface is reprojected onto the thermal images obtained by the thermal camera using the intrinsic camera parameters obtained during calibration, and if the point falls within the limits of the image, then that temperature is assigned to the point. The result 


\subsection{1/qirt.2016.020}

of this reconstruction is seen in figure 6 from three different views, however since the reconstruction is a 3D surface, it is possible to visualize it from any desired angle.
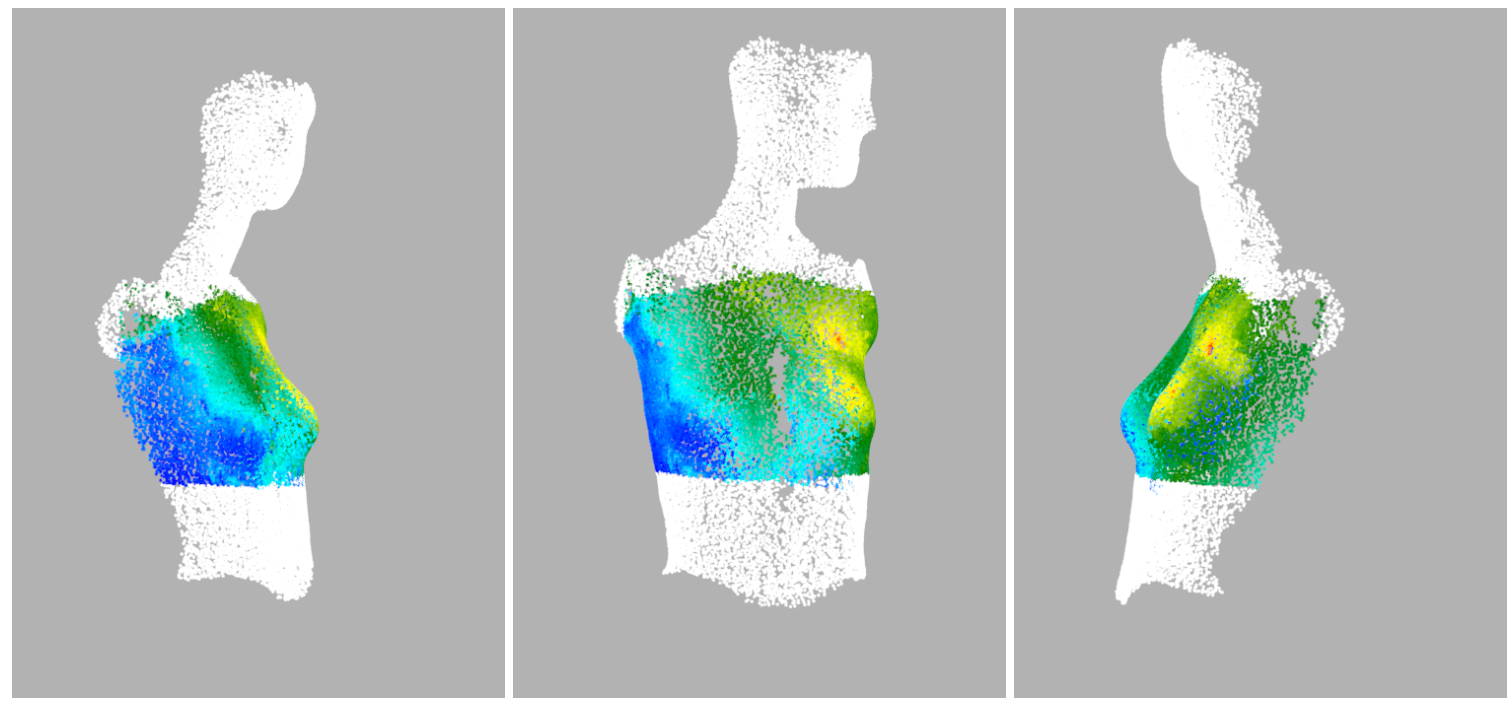

Fig. 6: Example of a 3D thermogram taken with the system, showing the reconstruction of the subject from different angles. Points that are white are not visible by the thermal cameras and thus have no temperature assigned to them.

\section{Conclusion}

The presence of 3D data about the surface under study has a lot of utility in research for biomedical applications. A big advantage of 3D data is the availability of information about the curvature and shape of the object, in particular, since the temperature shown by a thermal camera depends on the difference between the surface normal and the camera direction, having the surface normal means that it is possible to calculate the correct surface temperature at a given point on the surface, taking into account its curvature. Another advantage is the possibility to use the information about the shape of the subject to select a region of interest for further study taking into account the anatomy of the subject. Finally, it is possible to use the 3D data to simply eliminate the background from a 2D thermogram. While the presence of 3D data carries many advantages, working with more data carries additional costs and trade-offs. The major complication in working with 3D data as opposed to simple 2D images is that the required computing power grows exponentially. Additionally, due to the fact that depth images are noisy, there has to be a trade-off between accuracy and acquisition time, the more accurate one wants the 3D representation to be, the more depth images are needed to calculate said surface.

\section{Acknowledgements}

Authors gratefully acknowledge the financial support from CONACyT (Mexico) under grant 213208, from Convocatoria "Proyectos de desarrollo cientifco para atender problemas nacionales, 2013" and under grant 222496, from Convocatoria "Programa de Estímulos a la Innovación, 2015".

\section{REFERENCES}

[1] K. Ammer, "Thermography 2015-a computer-assisted literature survey, Thermology International," vol. 26, pp. 5-42, 2016.

[2] T. B. Borchartt, A. Conci, R. C. Lima, R. Resmini, and A. Sanchez, "Breast thermography from an image processing viewpoint: A survey," Signal Processing, vol. 93, p. 2785-2803, Oct 2013.

[3] S. E. Godoy, D. A. Ramirez, S. A. Myers, G. von Winckel, S. Krishna, M. Berwick, R. S. Padilla, P. Sen, and S. Krishna, "Dynamic infrared imaging for skin cancer screening," Infrared Physics \& Technology, vol. 70, pp. 147-152, 2015.

[4] S. Colantonio, G. Pieri, O. Salvetti, M. Benvenuti, S. Barone, and L. Carassale, "A method to integrate thermographic data and 3d shapes for diabetic foot disease," in Proceedings of 8th International Conference on Quantitative Infrared Thermography (QIRT), Padova (Italy), 2006.

[5] T.-Y. Cheng, D. Deng, and C. Herman, "Curvature effect quantificaiton for in-vivo IR thermography," Volume 2: Biomedical and Biotechnology, Nov 2012. 


\subsection{1/qirt.2016.020}

[6] Y. Chen and W. Warren, "3d fusion of infrared images with dense rgb reconstruction from multiple views-with application to fire-fighting robots," 2013.

[7] X. Ju, J.-C. Nebel, and J. P. Siebert, "3d thermography imaging standardization technique for inflammation diagnosis," in Photonics Asia 2004, pp. 266-273, International Society for Optics and Photonics, 2005.

[8] R. Yang and Y. Chen, "Design of a 3-d infrared imaging system using structured light," Instrumentation and Measurement, IEEE Transactions on, vol. 60, no. 2, pp. 608-617, 2011.

[9] I. Grubišić, "Medical 3d thermography system," Periodicum biologorum, vol. 113, no. 4, pp. 401-406, 2011.

[10] J. Sun, H. Ma, and D. Zeng, "Three-dimensional infrared imaging method based on binocular stereo vision," Opt. Eng, vol. 54, p. 103111, Oct 2015.

[11] H. Y.-M. Ng and R. Du, "Acquisition of 3d surface temperature distribution of a car body," in Information Acquisition, 2005 IEEE International Conference on, pp. 5-pp, IEEE, 2005.

[12] S. Prakash, P. Y. Lee, and T. Caelli, "3D mapping of surface temperature using thermal stereo," 2006 9th International Conference on Control, Automation, Robotics and Vision, 2006.

[13] K. Skala, T. Lipić, I. Sović, L. Gjenero, and I. Grubišić, "4d thermal imaging system for medical applications," Periodicum biologorum, vol. 113, no. 4, pp. 407-416, 2011.

[14] J. Rangel, S. Soldan, and A. Kroll, "3d thermal imaging: Fusion of thermography and depth cameras," in International Conference on Quantitative InfraRed Thermography, 2014.

[15] M. Kaczmarek and A. Nowakowski, "Active ir-thermal imaging in medicine," Journal of Nondestructive Evaluation, vol. 35, no. 1, pp. 1-16, 2016.

[16] L. Li, "Time-of-flight camera-an introduction," Technical White Paper, May, 2014.

[17] Y. Wang, Y. Li, and J. Zheng, "A camera calibration technique based on opencv," in Information Sciences and Interaction Sciences (ICIS), 2010 3rd International Conference on, pp. 403-406, IEEE, 2010.

[18] S. Paris, P. Kornprobst, J. Tumblin, and F. Durand, "A gentle introduction to bilateral filtering and its applications," in ACM SIGGRAPH 2007 courses, p. 1, ACM, 2007.

[19] R. B. Rusu and S. Cousins, "3d is here: Point cloud library (pcl)," in Robotics and Automation (ICRA), 2011 IEEE International Conference on, pp. 1-4, IEEE, 2011.

[20] M. Berger, A. Tagliasacchi, L. Seversky, P. Alliez, J. Levine, A. Sharf, and C. Silva, "State of the art in surface reconstruction from point clouds," in EUROGRAPHICS star reports, vol. 1, pp. 161-185, 2014. 